\title{
INDICE DE VOLÚMENES ANTERIORES
}

\section{Volumen XII (2)}

El Comportamiento del Mercado de Licores en el Departamento de Boyacá Carlos Julio Rodríguez Buitrago / Gladys Yaneth Mariño Becerra

La empresa y el Clima Organizacional

Luis Felipe Merchán

Una Aproximación al Análisis de la Competitividad Internacional de los Sectores Productivos

\section{Marlén Suarez Pineda}

El Administrador de Empresas y la Responsabilidad Tributaria para el Año 2012 Milton Orlando Burbano Galán

La Palabra y la Cultura en la Organización

Rosa Nelly Pérez Martínez

Responsabilidad Social, un nuevo componente a la luz de la Norma ISO 9001:2008

y la Guía GTC 180

Zonia Liliana Morales Ruíz

\section{Volumen XIII (1)}

El conocimiento administrativo, retos y oportunidades en la identificación de una epistemología de la administración

\section{Patricia Carolina Barreto Bernal}

Cultura organizacional como fuente de ventaja competitiva: el caso

de la empresa Arly en Tunja - Boyacá

Danithsa Vanesa Vega Avila

Lina Margarita Santos Cardozo

Teoría de la internalización y de los negocios internacionales: una visión desde la organización

Gina Paola Fonseca Cifuentes

Análisis del turismo en la Provincia de Sugamuxi departamento de Boyacá Ana Ruth Angarita Lizarazo

Karen Orozco Rodríguez

Experiencias en el uso de simuladores empresariales para la formación de administradores de empresas

Oscar Gutiérrez Molina

Interpretación administrativa de la novela "La Caverna" de José Saramago Jaime Ignacio Bermúdez Guerrero 
Volumen XIII (2)

\section{ADMINISTRACIÓN Y FINANZAS}

Efectos medioambientales en una contabilidad financiera dentro de una pyme, estudio de caso Omar García Jiménez / Claudia Yazmin Medina Contreras

Diagnóstico del área financiera del sector supermercados e hipermercados en el departamento de Boyacá.

Jorge Enrique Romero Muñoz / Diana Carolina Castelblanco Vargas / Martha Liliana Bastidas Arandia

Responsabilidad social empresarial en el sector minero de Colombia Jonathan Armando Bosigas Malagón / Jenny Edith Parada Camargo

Selección de los canales de distribución del ganado vacuno en Cumarál y Barranca de Upía

Martha Lucía Vargas Bacci / Marcos Javier Castelblanco Borja / Luis Enrique Masmela Casallas

\section{POLÍTICA Y SOCIAL}

El desplazamiento en Colombia; un flagelo, un estado de cosas inconstitucionales Juan Pablo Camargo Gómez

La Universidad Guadalajara y la igualdad de género. Propuesta Berta Ermila Madrigal Torres / Rosalba Madrigal Torres / Elia Marún Espinosa

El saber obrar en el mundo empresarial: por los caminos de la ética de mínimos Jhon Jairo Losada Cubillos

Volumen XIV (1)

\section{ADMINISTRACIÓN Y FINANZAS}

Competitividad sistémica en el sector agrícola: Una aproximación al concepto. Germán Andrés Monroy Medina / Marlén Suárez Pinéda Yemina Paola López

Efectos de la reforma tributaria 1607 del 2012 en el sector ganadero de Villavicencio. Fernando Baquero Cortes / Franzury Reyes Herrera Juan Diego Suarez Rojas

Responsabilidad Social y permanencia empresarial en pymes agrícolas: evidencia empírica en México

María De Los Ángeles Cervantes Rosas / Myrna Delfina López Noriega

Costos $\mathrm{ABC}$ para producción convencional de tomatede árbol en el departamento de Boyacá.

Diana Astrid Buitrago Núñez 


\section{MARKETING}

El E-Comerce como un medio para aumentar la productividaden las microempresas. Francisca Rosales Gómez / María Guadalupe Aguirre Alemán Oreste Pimentel Reyes / Daniel Ortiz Ventura

Un factor crítico para la gestión de la innovación tecnológica. Los símbolos de poder. Damaris Yunuen Mejía López

El comercio electrónico como herramienta de comercialización para los empresarios. Rosa María González López / Lilia Vázquez Martínez / Jorge Pérez Mejía

\section{Volumen XIV (2)}

\section{ADMINISTRACIÓN Y FINANZAS}

- Conocimiento del mercado de valores en las medianas y grandes empresas del departamento de Boyacá: diagnóstico y propuesta estratégica

Gina Paola Fonseca Cifuentes

Lady Johanna Castaño Buitrago

-Competitividad del sector transporte intermunicipal depasajeros en Boyacá.

Diego Armando Rodríguez Álvarez

Claudia Jessenia Becerra Gualdrón

-Gestión de la innovación en pequeñas empresas. Un estudio exploratorio en Querétaro.

Damaris Yunuen Mejía López

- Fórmula Du Pont y su rentabilidad, vista desde la óptica administrativa Omar García Jiménez

\section{MARKETING Y EDUCACIÓN}

- Mecanismos de influencia en el consumidor infantil de Villavicencio.

Laura Catalina Gallego Esquivel

Yehimi Carolina Higuera

María Cristina Otero Gómez

Wilson Giraldo Pérez

- La publicidad, una estrategia de éxito para las micro, pequeñas y medianas empresas de la región centro y suroestedel Estado de Hidalgo.

Ruth Flores Jiménez

Ivette Flores Jiménez

Raúl Rodríguez Moreno

Miguel Ángel Vázquez Alamilla

- Los valores en educación para el desarrollo.

Juan Manuel Zamora Mendoza

Miguel Ángel Medina Romero

Jean Cadet Odimba OnÉtambalako Wetshokonda 